\title{
Only one patient out of five achieves symmetrical knee function 6 months after primary anterior cruciate ligament reconstruction
}

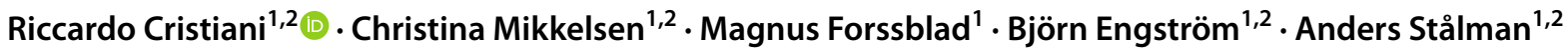

Received: 11 September 2018 / Accepted: 1 February 2019 / Published online: 18 February 2019

(c) The Author(s) 2019

\begin{abstract}
Purpose To assess the percentage of patients achieving symmetrical knee function 6 months after primary anterior cruciate ligament (ACL) reconstruction (ACLR) and to identify factors affecting its achievement, in a large cohort.

Methods Data were extracted from our clinic database. Patients who underwent primary ACLR from 2000 to 2015 and were assessed with the isokinetic quadriceps and hamstring muscles strength tests and single-leg-hop test at the 6-month follow-up were included in the study. Demographic data, information on the graft used, cartilage injuries and concomitant meniscal surgery were reviewed. Patients who reached a limb symmetry index (LSI) of $\geq 90 \%$ in all three tests were considered to have achieved symmetrical knee function. A multivariate logistic regression analysis was used to determine whether patient age, gender, time from injury to surgery, pre-injury Tegner activity level, graft type, cartilage injury and the presence of medial meniscus (MM) or lateral meniscus (LM) resection or repair were factors associated with the achievement of symmetrical knee function 6 months after primary ACLR.

Results A total of 4093 patients (54.3\% males) with a mean age of $28.3 \pm 10.7$ years were included. Data from all three tests were available for 3541 patients. The proportion of patients that achieved a LSI of $\geq 90 \%$ was $35.7 \%, 47.3 \%$ and $67.9 \%$ for isokinetic quadriceps muscle strength, hamstring muscles strength and the single-leg-hop test, respectively. A total of 693 patients (19.6\%) achieved symmetrical knee function, reaching a LSI of $\geq 90 \%$ in all three tests. Older age ( $\geq 30$ years) (OR, $0.50 ; 95 \%$ CI $0.41-0.61 ; P<0.001)$, MM resection (OR, $0.75 ; 95 \%$ CI $0.57-0.98 ; P=0.03)$ and MM repair (OR, $0.63 ; 95 \%$ CI 0.40-0.98; $P=0.04)$ reduced the odds, whereas the use of hamstring tendon (HT) autograft (OR, 2.28; 95\% CI 1.51-3.45; $P<0.001$ ) over bone-patellar tendon-bone (BPTB) autograft increased the odds of achieving symmetrical knee function.

Conclusion Only $19.6 \%$ of the patients achieved symmetrical knee function 6 months after primary ACLR. Age $\geq 30$ years, MM resection and MM repair reduced the chance, whereas the use of HT autograft over BPTB autograft increased the chance of achieving symmetrical knee function 6 months after primary ACLR. This study shows that most of the patients are yet to regain symmetrical knee function 6 months after primary ACLR and, moreover, it identifies several factors affecting its achievement in a large cohort. The results of this study should be used to counsel patients about their expected functional recovery and to optimize rehabilitation and maximize knee function after ACLR.
\end{abstract}

Level of evidence III.

Keywords Anterior cruciate ligament $\cdot$ ACL $\cdot$ Reconstruction $\cdot$ Quadriceps strength $\cdot$ Single leg hop test $\cdot$ Limb symmetry index $\cdot$ LSI $\cdot$ Rehabilitation $\cdot$ Knee function $\cdot$ Sports

Riccardo Cristiani

riccardo.cristiani87@gmail.com

Christina Mikkelsen

christina.mikkelsen@capio.se

Magnus Forssblad

magnus@forssblad.se

Björn Engström

bjorn.engstrom@capio.se
Anders Stålman

anders.stalman@ki.se

1 Department of Molecular Medicine and Surgery, Stockholm Sports Trauma Research Center, Karolinska Institutet, Stockholm, Sweden

2 Capio Artro Clinic, FIFA Medical Centre of Excellence, Sophiahemmet Hospital, Valhallavägen 91, 11486 Stockholm, Sweden 


\section{Introduction}

An anterior cruciate ligament (ACL) tear is a serious knee injury that can prevent patients from participating in both daily living and physical activities $[1,21]$. ACL reconstruction (ACLR) is common among patients who want to return to sport (RTS) [3, 18]. RTS is often advocated 6 months after ACLR [4]. However, this timeframe has recently been questioned, as the risk of sustaining an ACL re-injury is highest during the early period (6-12 months) of RTS $[8,17]$. Post-surgical time alone is not sufficient to determine readiness for RTS [8]. Functional tests, such as isokinetic muscle strength tests and the single-leg-hop test, are regarded as key factors prior to RTS [8, 34, 37]. The results of these tests are most frequently reported through the limb symmetry index (LSI) [34, 35]. The LSI is a measurement of the strength/function of the operated limb in relation to the healthy limb. The rationale is to ensure that the injured leg reaches an acceptable level of strength and function when returning to sport or strenuous activities. A LSI of $\geq 90 \%$ in these tests is the threshold value that should be reached for a successful and safe RTS $[9,13,21,31,34,43]$. Muscular asymmetries not only affect sport performance, they are also associated with a greater risk of ACL graft rupture and knee re-injuries after RTS $[8,17]$. Single-legged hop tests are able to discriminate between those individuals who return to their previous activity level from those who do not after ACLR [1]. Patients with symmetrical isokinetic muscle strength and hop performance 6 months after ACLR have superior knee function and higher activity levels at the midterm followup [31]. Therefore, rehabilitation should assist in restoring limb-to-limb symmetry.

To date, no previous studies have assessed the rate of patients achieving symmetrical knee function, measured as symmetry in isokinetic quadriceps and hamstring muscles strength and single-leg-hop test performance, 6 months after primary ACLR, in a large cohort. In addition, a detailed analysis of patients' factors affecting the achievement of symmetrical knee function has not previously been presented. The heterogeneity of patients' preoperative and intraoperative variables makes it difficult to predict which patients will be able to achieve symmetrical knee function 6 months after ACLR. However, this information would be very valuable for clinicians and physiotherapists to counsel patients about their expectations regarding functional recovery and to optimize rehabilitation and maximize knee function after ACLR.

The purpose of this study was to assess the percentage of patients achieving symmetrical knee function 6 months after primary ACLR and to identify factors affecting its achievement, in a large cohort. It was hypothesized that age, gender, graft choice, concomitant meniscal surgery and cartilage injuries would affect the chance of achieving symmetrical knee function at the 6-month follow-up.

\section{Materials and methods}

Data were extracted from our clinic database. A total of 4341 patients who underwent primary ACLR from 2000 to 2015 and were assessed at the 6-month follow-up with the isokinetic quadriceps and hamstring muscles strength tests and the single-leg-hop test were identified. All the included patients had no concomitant ligament injuries. Patients who had contralateral ACL injuries or reconstructions $(n=248)$ were excluded.

\section{Surgical technique and rehabilitation}

All the patients were operated on using a single-bundle autologous hamstring tendon (HT) or bone-patellar tendon-bone (BPTB) technique. For the ACLRs performed with HT graft, the semitendinosus tendon was primarily harvested and prepared as a triple or quadruple graft. If the length or the diameter of the graft was considered insufficient $(<8 \mathrm{~mm})$, the gracilis tendon was additionally harvested and combined with the semitendinosus graft. The BPTB graft was harvested as the central third of the patellar tendon with two bone blocks. Both grafts were routinely fixed using an Endobutton fixation device (Smith \& Nephew, Andover, Mass, USA) on the femoral side and Ethibond no. 2 sutures (Ethicon, Sommerville, NJ) tied over an AO bicortical screw with a washer as a post or using an interference screw on the tibial side. Meniscal repair was performed, for both the medial meniscus (MM) and lateral meniscus (LM), with an arthroscopic all-inside technique using a Fast-Fix suture anchor device (Smith and Nephew, Andover, Mass, USA) or an inside-out technique for tears located in the dorsal and middle portion of the meniscus. An outside-in technique was used for tears located in the anterior portion of the meniscus. Both inside-out and outside-in meniscal repair techniques were performed using PDS 0 (Ethicon, Sommerville, NJ). All the patients followed a standardized postoperative rehabilitation protocol. In the event of an isolated ACLR or an ACLR with simultaneous meniscal resection, full weight bearing and full range of motion were encouraged as tolerated. If a meniscal repair was performed, patients wore a hinged knee brace for 6 weeks. Flexion was limited from $0^{\circ}$ to $30^{\circ}$ for the first 2 weeks, from $0^{\circ}$ to $60^{\circ}$ for the third and fourth weeks, and from $0^{\circ}$ to $90^{\circ}$ for the fifth and sixth weeks after surgery. Starting from the seventh week, the knee brace was discontinued and progressive weight bearing was allowed. The early rehabilitation phase focused on regaining range of motion, reduction of swelling 
and gait correction. The rehabilitation protocol included joint and muscle flexibility exercises, balance/coordination training and strength training focusing primarily on the thigh muscles. For all patients, quadriceps strengthening was restricted to closed kinetic chain exercises during the first 3 months. On the basis of muscle strength, functional hop performance and type of sport, the patients were allowed to RTS 6 months postoperatively at the earliest.

\section{Outcome}

All the patients were evaluated at our outpatient clinic 6 months postoperatively, using a standardized protocol. Isokinetic concentric quadriceps and hamstring muscles strength was measured bilaterally at $90^{\circ} \mathrm{s}$ using the Biodex System 3 (Biodex Medical Systems, Shirley, New York, USA) [6]. The test was performed in a range of motion between $90^{\circ}$ and $10^{\circ}$ of knee flexion, always starting with the contralateral uninjured knee. Prior to the test, the patients warmed up using a stationary cycling ergometer at low resistance for $10 \mathrm{~min}$. Patients were given a verbal description of the test and two-three practical trials were allowed before testing. Each patient performed five maximal quadriceps and hamstring muscles contractions with each leg. Patients were verbally encouraged during the test. The peak quadriceps and hamstring torque values (highest achieved values) were registered.

The single-leg-hop test was used to assess functional hop performance [25, 27]. The test was performed with the patient standing on one leg and being instructed to jump straight ahead as far as possible and land on the same leg. The test was considered successful if the landing was stable. If the patient landed with an early touchdown of the contralateral limb, had a loss of balance or took additional hops after landing, the hop was repeated. Patients were initially given a verbal description of the test and they were allowed to perform as many practical trials as they wanted, until they felt confident about the test. Three trials were performed for each leg, always starting with the contralateral uninjured leg [2]. Patients were given as much time as they wanted between the trials to minimize fatigue. The best trial for each leg was registered. Patients who did not want to undertake the test, who did not feel confident enough about the test or who did not undertake jumping exercises during the rehabilitation were not tested.

All three tests (isokinetic quadriceps and hamstring muscles strength tests and single-leg-hop test) were conducted by experienced sports medicine physiotherapists who had undergone extensive training in these testing procedures. The limb symmetry indexes (LSIs) of the peak quadriceps and hamstring muscles torque and single-leg-hop test were calculated as [involved limb/uninvolved limb $\times 100$ ] for each test. The achievement of a symmetrical isokinetic muscle strength or single leg hop test performance was defined as performing at least $90 \%$ of the contralateral leg (LSI $\geq 90 \%)$ $[9,24,34]$. Patients who reached a LSI of $\geq 90 \%$ in all three tests were considered to have achieved symmetrical knee function.

\section{Data sources}

Demographic data (age and gender), information about the time from injury to surgery, pre-injury Tegner activity level [32], graft type, meniscus surgery and the presence of cartilage injuries were collected in our local database. Meniscus surgery was classified as follows: no meniscus surgery, meniscus resection, or meniscus repair for both medial and lateral meniscus. The results of the isokinetic quadriceps and hamstring muscles strength tests and the single-leg-hop test 6 months following ACLR were reviewed.

Ethical permission for this study was obtained from the regional ethics committee, Karolinska Institutet (Diarienumber 2016/1613-31/32).

\section{Statistical analysis}

The Statistical Package for Social Sciences, SPSS (Version 25.0, IBM Corp., Armonk, New York, USA) was used for the statistics. All variables were summarized with standard descriptive statistics such as mean, standard deviations, or frequency. Symmetrical isokinetic quadriceps or hamstring muscles strength and single-leg-hop test performance were defined as achieving a LSI of $\geq 90 \%$ ("Yes" vs. "No") in each test and were used as outcome measure. The relationship between the three outcome measures (quadriceps muscle strength, hamstring muscles strength and single-leg-hop test performance) was investigated using the phi-coefficient. Three separate multivariate logistic regression analysisisokinetic quadriceps muscle strength, hamstring muscles strength, single-leg-hop test performance-were performed with age, gender (female vs. male), time from injury to surgery (delayed $>3$ months vs. not delayed $\leq 3$ months), preinjury Tegner activity level (high $\geq 6$ vs. low $<6$ ), graft (HT vs. BPTB autograft), medial meniscus resection, medial meniscus repair, lateral meniscus resection, lateral meniscus repair, and cartilage injury as independent variables and the achievement of symmetrical (LSI $\geq 90 \%$ ) isokinetic quadriceps muscle strength, hamstring muscles strength and single-leg-hop test performance as dependent variables. An additional multivariate logistic regression analysis was performed with the same independent variables and the achievement of a LSI of $\geq 90 \%$ in all three tests (symmetrical knee function) as the dependent variable. Age was dichotomized into classes close to the median ( $\geq 30$ years vs. $<30$ years). The relationships were expressed as odds ratios (OR) with 
95\% confidence intervals (CI). The level of significance in all analysis was 5\% (two-tailed).

Provided a significance level of $5 \%$, a power of $85 \%$, and a sample size of more than 1000 patients, even a very weak relationship of less than 0.10 (phi coefficient), which corresponds to an effect size of less than 0.10 according to Cohen, would be detected.

\section{Results}

A total of 4,093 patients, with 6-month isokinetic quadriceps and hamstring muscles strength tests data, formed the study cohort. Of these, data for the single-leg-hop test were available for 3541 patients. Patient characteristics are summarized in Table 1.

The relationship between the three outcome measures is shown in Table 2.

\section{Isokinetic quadriceps muscle strength}

A total of 1463 patients $(35.7 \%)$ in the studied cohort achieved symmetrical isokinetic quadriceps muscle strength. Age $\geq 30$ years, odds ratio [OR] 0.47; 95\% confidence interval [CI] 0.41-0.55; $P<0.001$ ), female gender (OR, 0.80; 95\% CI $0.70-0.91 ; P=0.001), \mathrm{MM}$ repair (OR, 0.60; $95 \%$ CI $0.43-0.84 ; P=0.003$ ) and LM repair (OR, $0.61 ; 95 \% \mathrm{CI}$ $0.42-0.89 ; P=0.01)$ reduced the odds, whereas the use of HT autograft (OR, 3.41; 95\% CI 2.48-4.69; $P<0.001)$ over

Table 1 Patient characteristics ( $n=4093$ Patients)

\begin{tabular}{|c|c|c|c|c|c|c|c|}
\hline & & \multicolumn{2}{|c|}{ Quadriceps strength ratio } & \multicolumn{2}{|c|}{ Hamstring strength ratio } & \multicolumn{2}{|c|}{ Single-leg-hop test ratio ${ }^{a}$} \\
\hline & & Mean \pm SD & LSI $\geq 90 \%$ & Mean \pm SD & LSI $\geq 90 \%$ & Mean \pm SD & LSI $\geq 90 \%$ \\
\hline \multicolumn{8}{|l|}{ Preoperative factors } \\
\hline Age at surgery, years, mean \pm SD & $28.3 \pm 10.7$ & & & & & & \\
\hline Aged younger than 30 years & $20.7 \pm 4.7 ; 2408(58.8)$ & $86.5 \pm 14.8$ & $992(41.2)$ & $91.0 \pm 22.4$ & $1194(49.6)$ & $94.2 \pm 10.8$ & $1579(72.7)$ \\
\hline Aged 30 years or older & $39.1 \pm 6.9 ; 1685(41.2)$ & $81.5 \pm 16.4$ & $445(26.4)$ & $88.6 \pm 15.4$ & $741(44.0)$ & $90.2 \pm 15.0$ & $828(60.4)$ \\
\hline \multicolumn{8}{|l|}{ Gender } \\
\hline Male & $2223(54.3)$ & $84.8 \pm 15.9$ & $804(36.2)$ & $90.7 \pm 22.8$ & $1078(48.5)$ & $93.4 \pm 12.2$ & $1377(71.5)$ \\
\hline Female & $1870(45.7)$ & $84.0 \pm 15.4$ & $633(33.8)$ & $89.2 \pm 15.6$ & $856(45.8)$ & $91.7 \pm 13.5$ & $1029(63.7)$ \\
\hline $\begin{array}{l}\text { Time from injury to surgery, } \\
\text { months, mean } \pm \text { SD }\end{array}$ & $16.9 \pm 29.8$ & & & & & & \\
\hline$\leq 3$ months & $646(15.8)$ & $84.0 \pm 15.2$ & $234(36.2)$ & $91.1 \pm 12.6$ & $342(52.9)$ & $94.4 \pm 10.0$ & $439(75.8)$ \\
\hline$>3$ months & $3447(84.2)$ & $84.6 \pm 15.6$ & $1202(34.9)$ & $89.6 \pm 16.0$ & $1593(46.2)$ & $92.3 \pm 13.3$ & $1967(66.4)$ \\
\hline $\begin{array}{l}\text { Pre-injury Tegner activity level, } \\
\text { median (range) }\end{array}$ & $7(1-10)$ & & & & & & \\
\hline High, $\geq 6$ & $3618(88.4)$ & $84.7 \pm 15.2$ & $1286(35.6)$ & $90.0 \pm 15.2$ & $1733(47.9)$ & $93.1 \pm 12.6$ & $2208(69.7)$ \\
\hline Low, $<6$ & 475 (11.6) & $82.7 \pm 17.8$ & $150(31.6)$ & $88.5 \pm 17.8$ & $206(43.4)$ & $88.8 \pm 13.9$ & $199(53.3)$ \\
\hline \multicolumn{8}{|l|}{ Intraoperative factors } \\
\hline \multicolumn{8}{|l|}{ Graft type } \\
\hline HT autograft & $3788(92.5)$ & $85.1 \pm 15.2$ & $1387(36.6)$ & $89.3 \pm 15.3$ & $1726(45.6)$ & $92.8 \pm 12.7$ & $2267(68.8)$ \\
\hline BPTB autograft & $305(7.5)$ & $76.1 \pm 17.1$ & $49(16.1)$ & $96.6 \pm 16.8$ & $209(68.5)$ & $89.9 \pm 13.4$ & $141(57.5)$ \\
\hline No meniscus surgery & $2577(63.0)$ & $84.7 \pm 15.9$ & $906(35.2)$ & $89.9 \pm 15.1$ & $1210(47.0)$ & $93.0 \pm 12.8$ & $1502(58.3)$ \\
\hline \multicolumn{8}{|l|}{ Medial meniscus surgery } \\
\hline Resection & $578(14.1)$ & $84.0 \pm 14.8$ & $178(30.8)$ & $88.7 \pm 15.3$ & $258(44.6)$ & $90.3 \pm 13.9$ & $298(60.9)$ \\
\hline Repair & $194(4.7)$ & $81.9 \pm 15.6$ & $53(27.3)$ & $89.7 \pm 13.9$ & $92(47.4)$ & $91.6 \pm 11.4$ & $105(61.4)$ \\
\hline \multicolumn{8}{|l|}{ Lateral meniscus surgery } \\
\hline Resection & $594(14.5)$ & $84.2 \pm 13.9$ & $208(35.0)$ & $89.5 \pm 13.9$ & $281(47.3)$ & $93.1 \pm 10.7$ & $364(61.3)$ \\
\hline Repair & $150(3.7)$ & $82.3 \pm 14.4$ & $43(28.7)$ & $92.6 \pm 20.6$ & $71(47.3)$ & $92.8 \pm 16.6$ & $80(62.5)$ \\
\hline \multicolumn{8}{|l|}{ Cartilage injury } \\
\hline Yes & $738(18.3)$ & $82.3 \pm 16.6$ & $222(30.1)$ & $88.3 \pm 14.9$ & 308 (41.7) & $90.5 \pm 14.4$ & $362(60.4)$ \\
\hline No & 3355 (81.7) & $84.9 \pm 15.3$ & $1214(36.2)$ & $90.2 \pm 15.7$ & 1631 (48.6) & $93.1 \pm 12.4$ & 2044 (69.5) \\
\hline
\end{tabular}

Data are reported as $\mathrm{n}(\%)$, unless otherwise indicated

$H T$ hamstring tendon, BPTB bone-patellar tendon-bone, $L S I$ limb symmetry index, $S D$ standard deviation

${ }^{\text {a }}$ Data available for 3541 patients 
Table 2 Relationships between the outcome measures investigated with the Phi coefficient

\begin{tabular}{llll}
\hline Outcome variable & $\begin{array}{l}\text { Quadriceps } \\
\text { strength } \\
\text { LSI } \geq 90 \%\end{array}$ & $\begin{array}{l}\text { Hamstring } \\
\text { strength } \\
\text { LSI } \geq 90 \%\end{array}$ & $\begin{array}{l}\text { Single- } \\
\text { leg-hop } \\
\text { test } \\
\text { LSI } \geq 90 \%\end{array}$ \\
\hline $\begin{array}{l}\text { Quadriceps strength } \\
\text { LSI } \geq 90 \%\end{array}$ & - & $0.22^{\mathrm{a}}$ & $0.26^{\mathrm{a}}$ \\
$\begin{array}{l}\text { Hamstring strength } \\
\text { LSI } \geq 90 \%\end{array}$ & - & $0.11^{\mathrm{b}}$ \\
$\begin{array}{l}\text { Single-leg-hop test } \\
\text { LSI } \geq 90 \%\end{array}$ & & & \\
\hline
\end{tabular}

LSI limb symmetry index

${ }^{\mathrm{a}}$ Weak positive correlation (Phi coefficient between 0.20 and 0.29 )

${ }^{\mathrm{b}}$ No or negligible correlation (Phi coefficient between 0.01 and 0.19 )

BPTB autograft increased the odds of achieving symmetrical quadriceps muscle strength. No correlation was found between the achievement of a LSI of $\geq 90 \%$ for quadriceps muscle strength and the time from injury to surgery, preinjury Tegner activity level, cartilage injury and MM or LM resection (Table 3).

\section{Isokinetic hamstring muscles strength}

The total number of patients who achieved symmetrical isokinetic hamstring muscles strength was 1,935 (47.3\%). Age $\geq 30$ years $(\mathrm{OR}, 0.85 ; 95 \%$ CI $0.74-0.97 ; P=0.02)$, delayed ( $>3$ months) ACLR (OR, 0.81; 95\% CI 0.68-0.97; $P=0.02)$, HT autograft (OR, $0.40 ; 95 \%$ CI $0.31-0.51$; $P<0.001)$ and cartilage injury (OR, 0.82; 95\% CI 0.69-0.97; $P=0.02$ ) reduced the odds of achieving symmetrical hamstring muscles strength. No other preoperative or intraoperative patient factors were found to be associated with the achievement of a LSI of $\geq 90 \%$ for hamstring muscles strength (Table 4).

\section{Single-leg-hop test performance}

The patients who achieved a symmetrical performance in the operated limb in relation to the healthy limb for the single-leg-hop test were $2,405(67.9 \%)$. Age $\geq 30$ years (OR, 0.60 ; 95\% CI, 0.51-0.70; $P<0.001$ ), female gender (OR, $0.64 ; 95 \%$ CI $0.55-0.75 ; P<0.001)$, delayed ( $>3$ months) ACLR (OR, 0.70; 95\% CI 0.57-0.87; $P<0.001$ ), MM resection (OR, $0.73 ; 95 \%$ CI $0.60-0.90 ; P=0.003$ ), MM repair (OR, $0.71 ; 95 \%$ CI $0.51-0.99 ; P=0.04)$ and cartilage injury (OR, $0.78 ; 95 \%$ CI $0.65-0.95 ; P=0.01$ ) reduced the odds, whereas high pre-injury Tegner activity level $(\geq 6)(\mathrm{OR}$, 1.59 ; $95 \%$ CI $1.26-1.99 ; P<0.001)$ and the use of HT autograft (OR 1.95; 95\% CI 1.48-2.56; $P<0.001$ ) over BPTB autograft increased the odds of achieving a symmetrical single-leg-hop test performance. No correlation was found between the achievement of a LSI of $\geq 90 \%$ for the singleleg-hop test and LM resection or repair (Table 5).

\section{Symmetrical knee function}

A total of 693 patients (19.6\%) achieved symmetrical knee function, reaching a LSI of $\geq 90 \%$ in all three tests (isokinetic quadriceps and hamstring muscles strength tests and single-leg-hop test). Age $\geq 30$ years (OR, 0.50; 95\% CI $0.41-0.61 ; P<0.001)$, MM resection (OR, $0.75 ; 95 \% \mathrm{CI}$, $0.57-0.98 ; P=0.03)$ and MM repair (OR, $0.63 ; 95 \% \mathrm{CI}$ $0.40-0.98 ; P=0.04)$ reduced the odds, whereas the use of HT autograft (OR, $2.28 ; 95 \%$ CI $1.51-3.45 ; P<0.001)$ over BPTB autograft increased the odds of achieving symmetrical knee function (Table 6).
Table 3 Factors associated with the achievement of symmetrical (LSI $\geq 90 \%$ ) isokinetic quadriceps muscle strength after ACL reconstruction in multivariate logistic regression analysis ( $n=4093$ Patients)

\begin{tabular}{lllll}
\hline Factor & $\begin{array}{l}\text { Regression coef- } \\
\text { ficient }(\beta)\end{array}$ & SE & O.R. (95\% CI) & $P$ value \\
\hline Age $\geq 30$ years & -0.73 & 0.07 & $0.47(0.41-0.55)$ & $<0.001^{*}$ \\
Female gender & -0.22 & 0.06 & $0.80(0.70-0.91)$ & $0.001^{*}$ \\
Delayed (>3 months) ACLR & 0.05 & 0.09 & $1.05(0.87-1.26)$ & $\mathrm{ns}$ \\
Pre-injury Tegner activity level $\geq 6$ & -0.06 & 0.11 & $0.93(0.75-1.16)$ & $\mathrm{ns}$ \\
HT autograft & 1.22 & 0.16 & $3.41(2.48-4.69)$ & $<0.001^{*}$ \\
MM resection & -0.16 & 0.10 & $0.84(0.69-1.02)$ & $\mathrm{ns}$ \\
MM repair & -0.49 & 0.16 & $0.60(0.43-0.84)$ & $0.003^{*}$ \\
LM resection & -0.05 & 0.09 & $0.94(0.78-1.14)$ & $\mathrm{ns}$ \\
LM repair & -0.48 & 0.18 & $0.61(0.42-0.89)$ & $0.01^{*}$ \\
Cartilage injury & -0.09 & 0.09 & $0.91(0.76-1.09)$ & $\mathrm{ns}$ \\
\hline
\end{tabular}

$L S I$ limb symmetry index, $A C L$ anterior cruciate ligament, $A C L R$ anterior cruciate ligament reconstruction, $H T$ hamstring tendon, $M M$ medial meniscus, $L M$ lateral meniscus, $S E$ standard error, $O R$ odds ratio, $C I$ confidence interval

*Statistically significant. $P$ value $<0.05$ 
Table 4 Factors associated with the achievement of symmetrical (LSI $\geq 90 \%$ ) isokinetic hamstring muscles strength after ACL reconstruction in multivariate logistic regression analysis $(n=4093$ Patients)
Table 5 Factors associated with the achievement of symmetrical (LSI $\geq 90 \%$ ) single leg hop test performance after ACL reconstruction in multivariate logistic regression analysis $(n=3541$ Patients)
Table 6 Factors associated with the achievement of symmetrical knee function (LSI $\geq 90 \%$ for isokinetic quadriceps and hamstring muscles strength and single-leg-hop test performance) after ACL reconstruction in multivariate logistic regression analysis ( $n=3541$ Patients)

\begin{tabular}{lllll}
\hline Factor & $\begin{array}{l}\text { Regression coef- } \\
\text { ficient }(\beta)\end{array}$ & SE & OR (95\% CI) & $P$ value \\
\hline Age $\geq 30$ years & -0.15 & 0.06 & $0.85(0.74-0.97)$ & $0.02 *$ \\
Female gender & -0.08 & 0.06 & $0.91(0.80-1.04)$ & $\mathrm{ns}$ \\
Delayed (>3 months) ACLR & -0.20 & 0.08 & $0.81(0.68-0.97)$ & $0.02^{*}$ \\
Pre-injury Tegner activity level $\geq 6$ & 0.11 & 0.10 & $1.12(0.91-1.37)$ & $\mathrm{ns}$ \\
HT autograft & -0.90 & 0.12 & $0.40(0.31-0.51)$ & $<0.001^{*}$ \\
MM resection & -0.04 & 0.09 & $0.95(0.79-1.14)$ & $\mathrm{ns}$ \\
MM repair & 0.02 & 0.15 & $1.02(0.76-1.37)$ & $\mathrm{ns}$ \\
LM resection & -0.02 & 0.09 & $0.97(0.81-1.16)$ & $\mathrm{ns}$ \\
LM repair & -0.03 & 0.17 & $0.96(0.69-1.34)$ & $\mathrm{ns}$ \\
Cartilage injury & -0.19 & 0.08 & $0.82(0.69-0.97)$ & $0.02^{*}$ \\
\hline
\end{tabular}

$L S I$ limb symmetry index, $A C L$ anterior cruciate ligament, $A C L R$ anterior cruciate ligament reconstruction, $H T$ hamstring tendon, $M M$ medial meniscus, $L M$ lateral meniscus, $S E$ standard error, $O R$ odds ratio, $C I$ confidence interval

*Statistically significant. $P$ value $<0.05$

\begin{tabular}{lllll}
\hline Factor & $\begin{array}{l}\text { Regression coef- } \\
\text { ficient }(\beta)\end{array}$ & SE & OR (95\% CI) & $P$ value \\
\hline Age $\geq 30$ years & -0.51 & 0.07 & $0.60(0.51-0.70)$ & $<0.001^{*}$ \\
Female gender & -0.43 & 0.07 & $0.64(0.55-0.75)$ & $<0.001^{*}$ \\
Delayed (>3 months) ACLR & -0.34 & 0.10 & $0.70(0.57-0.87)$ & $<0.001^{*}$ \\
Pre-injury Tegner activity level $\geq 6$ & 0.46 & 0.11 & $1.59(1.26-1.99)$ & $<0.001^{*}$ \\
HT autograft & 0.66 & 0.13 & $1.95(1.48-2.56)$ & $<0.001^{*}$ \\
MM resection & -0.30 & 0.10 & $0.73(0.60-0.90)$ & $0.003^{*}$ \\
MM repair & -0.34 & 0.17 & $0.71(0.51-0.99)$ & $0.04^{*}$ \\
LM resection & 0.10 & 0.10 & $1.11(0.90-1.38)$ & $\mathrm{ns}$ \\
LM repair & -0.38 & 0.19 & $0.68(0.47-1.00)$ & $\mathrm{ns}$ \\
Cartilage injury & -0.24 & 0.09 & $0.78(0.65-0.95)$ & $0.01^{*}$ \\
\hline
\end{tabular}

$L S I$ limb symmetry index, $A C L$ anterior cruciate ligament, $A C L R$ anterior cruciate ligament reconstruction, $H T$ hamstring tendon, $M M$ medial meniscus, $L M$ lateral meniscus, $S E$ standard error, $O R$ odds ratio, $C I$ confidence interval

*Statistically significant. $P$ value $<0.05$

\begin{tabular}{lllll}
\hline Factor & $\begin{array}{l}\text { Regression coef- } \\
\text { ficient }(ß)\end{array}$ & SE & OR (95\% CI) & $P$ value \\
\hline Age $\geq 30$ years & -0.68 & 0.09 & $0.50(0.41-0.61)$ & $<0.001^{*}$ \\
Female gender & -0.14 & 0.08 & $0.86(0.72-1.02)$ & $\mathrm{ns}$ \\
Delayed (>3 months) ACLR & -0.11 & 0.11 & $0.89(0.71-1.11)$ & $\mathrm{ns}$ \\
Pre-injury Tegner activity level $\geq 6$ & 0.04 & 0.15 & $1.04(0.77-1.40)$ & $\mathrm{ns}$ \\
HT autograft & 0.82 & 0.21 & $2.28(1.51-3.45)$ & $<0.001^{*}$ \\
MM resection & -0.29 & 0.13 & $0.75(0.57-0.98)$ & $0.03^{*}$ \\
MM repair & -0.45 & 0.22 & $0.63(0.40-0.98)$ & $0.04^{*}$ \\
LM resection & 0.05 & 0.12 & $1.06(0.83-1.34)$ & $\mathrm{ns}$ \\
LM repair & -0.30 & 0.24 & $0.73(0.45-1.18)$ & $\mathrm{ns}$ \\
Cartilage injury & -0.15 & 0.12 & $0.86(0.67-1.10)$ & $\mathrm{ns}$ \\
\hline
\end{tabular}

$L S I$ limb symmetry index, $A C L$ anterior cruciate ligament, $A C L R$ anterior cruciate ligament reconstruction, $H T$ hamstring tendon, $M M$ medial meniscus, $L M$ lateral meniscus, $S E$ standard error, $O R$ odds ratio, $C I$ confidence interval

*Statistically significant. $P$ value $<0.05 \mathrm{~s}$ 


\section{Discussion}

The most important finding of the present study was that only $19.6 \%$ of the patients achieved symmetrical knee function 6 months after ACLR, reaching a LSI of $\geq 90 \%$ in all three tests. The proportion of patients that achieved a LSI of $\geq 90 \%$ for isokinetic quadriceps muscle strength, hamstring muscles strength and the single-leg-hop test was $35.7 \%$, $47.3 \%$ and $67.9 \%$, respectively.

\section{Isokinetic quadriceps muscle strength}

In the analysis of quadriceps muscle strength, age $\geq 30$ years, female gender, MM repair and LM repair reduced the odds, whereas the use of HT autograft over BPTB autograft increased the odds of achieving symmetrical muscle strength. In a recent study, Ueda et al. [36] showed that older age and female gender are negatively associated with quadriceps strength recovery 6 months after ACLR. Iriuchishima et al. [12] also reported that older age is associated with residual muscle weakness 9 months after ACLR. These findings highlight that age- and gender-specific rehabilitation programs, with greater emphasis on quadriceps strength training for older and female patients, should be considered to facilitate strength recovery after ACLR.

Interestingly, patients who underwent a concomitant MM or LM repair had a lower odds of achieving symmetrical quadriceps muscle strength 6 months after ACLR. This is probably related to the fact that they underwent a different rehabilitation regime, with delayed onset of full weightbearing and strength training due to the use of a postoperative hinged knee brace for the first 6 weeks after ACLR. As expected, graft choice between BPTB and HT autograft strongly affected the opportunity to achieve symmetrical quadriceps muscle strength. The use of HT autograft instead of BPTB autograft was found to be a factor strongly associated (OR, 3.41; 95\% CI 2.48-4.69; $P<0.001$ ) with the recovery of quadriceps muscle strength. Graft harvesting is accompanied by donor-site morbidity and muscle function impairment. A systematic review and meta-analysis [41] has shown that muscle weakness after ACLR is dependent on the graft donor site. Patients with HT autograft experience increased strength deficits with knee flexion, while patients with BPTB autograft experience increased strength deficits with knee extension.

\section{Isokinetic hamstring muscles strength}

Age $\geq 30$ years, delayed ( $>3$ months) ACLR, HT autograft and cartilage injury were found to be associated with a decreased odds of achieving symmetrical hamstring muscles strength, 6 months after ACLR. In a recent study, Krych et al. [16], reported that younger age and minimal cartilage degeneration are associated with excellent isokinetic muscle strength (LSI $\geq 85 \%$ ) and functional hop performance (LSI $\geq 90 \%) 6$ months after primary ACLR. Again, reduced muscle strength was found to be strongly correlated with the harvest site. For HT autograft ACLR, there has always been concern about hamstring weakness [15, 42]. It has been shown that HT autograft harvesting can reduce hamstring muscles strength for up to 1-2 years after ACLR [28]. Interestingly, time from injury to surgery longer than 3 months significantly reduced the odds of achieving symmetrical hamstring muscles strength. It can be hypothesized that, during the preoperative rehabilitation, more emphasis is generally placed on quadriceps muscle strengthening, resulting in a possible deconditioning of the hamstring muscles, in the event of a longer time interval from injury to surgery.

\section{Single-leg-hop test performance}

Single-legged hop tests are performance-based measures used to assess the combination of muscle strength, neuromuscular control, confidence in the limb and the ability to tolerate loads related to sport-specific activities [20]. The single-leg-hop test is consistently reported in the literature to quantify knee performance after ACLR [10, 25, 40]. In the analysis of single-leg-hop test performance, age $\geq 30$ years, female gender, delayed ( $>3$ months) ACLR, MM resection or MM repair and cartilage injury were found to be negatively associated with the opportunity to achieve a LSI of $\geq 90 \%$ six months after ACLR. Again, these findings highlight the fact that more attention should be paid to older and female patients. These categories of patients could need more specific rehabilitation programs after ACLR. As reported for the hamstring muscles strength analysis, a time from injury to surgery longer than 3 months was found to be associated with a lower opportunity to achieve a symmetrical single-leg-hop test performance. This could be due to the potential deconditioning of the injured knee over time. Patients with an ACL injury could experience a feeling of knee instability that could prevent their participation in physical activities, leading to the progressive deterioration of their knee function over time. Our study also revealed that the presence of MM resection, MM repair or cartilage injury was associated with a reduced odds of achieving limb symmetry for this test. This finding could be expected, as the presence of meniscal or cartilage injuries can cause knee pain [28], thereby resulting in a poorer single-leg-hop test performance. A pre-injury Tegner activity level $\geq 6$ and the use of HT autograft over BPTB autograft were the two factors that increased the odds of achieving a symmetrical single-leg-hop test performance 6 months after ACLR. For the HT autograft ACLR, less anterior knee pain and kneeling 
pain than the BPTB autograft ACLR have been reported at a short-term follow-up [36]. These, together with the greater quadriceps strength associated with the use of this graft compared with BPTB autograft, could be responsible for the superior 6-month single-leg-hop test performance after ACLR performed with HT autograft. Patients with a high pre-injury Tegner activity level could begin rehabilitation with better knee function, be more eager to RTS, and therefore, spend more time and efforts in the rehabilitation process than patients with a low pre-injury activity level.

\section{Symmetrical knee function}

When the effect of the independent variables on the achievement of a LSI $\geq 90 \%$ in all three tests (symmetrical knee function) was analyzed in our multivariate logistic regression analysis, it was found that age $\geq 30$ years, MM resection and MM repair were the factors that reduced the odds, whereas the use of HT autograft over BPTB autograft was the only factor that increased the odds of achieving symmetrical knee function 6 months after ACLR.

It is accepted that muscle loss occurs with aging [30]. There is a link between age and loss of muscle fiber number and size [26]. However, the results of our study were expressed for both isokinetic muscle strength and single-leghop test performance as LSIs, comparing the ACL-reconstructed knee with the healthy knee. A muscle loss with aging could also be expected in the healthy knee, thereby not influencing the LSI. It is possible that, for older patients, the reduced odds of achieving symmetry for isokinetic muscle strength and single-leg-hop test performance could be related to a reduction in physical activities. Older patients could have lower knee demands compared with younger patients and they could, therefore, be less engaged in the rehabilitation process. The effect of a concomitant MM resection and MM repair on reducing the odds of achieving symmetrical isokinetic quadriceps muscle strength and single-leg-hop test performance has already been examined. These concurrent surgical procedures were still negatively associated with the opportunity to achieve a LSI of $\geq 90 \%$ in all three tests 6 months after ACLR, in our multivariate logistic regression analysis. The Graft choice between BPTB and HT autograft strongly influenced the likelihood of achieving symmetrical knee function six months after ACLR. A 2.28-fold (95\% CI 1.51-3.45; $P<0.001)$ increase was found in the odds of achieving symmetrical knee function for patients receiving HT autograft. Although this finding could be interpreted as promoting the use of HT autograft over BPTB autograft for the earlier achievement of symmetrical knee function and RTS, it should be interpreted with caution. The ligamentization of the ACL graft occurs over a period of 1-2 years after surgery $[5,29]$ and the risk of sustaining an ACL re-injury is greatest during the early period (6-12 months) of RTS [8,
17]. Recent studies from the Scandinavian ACL registries [7, 23] reported an increased risk of revision with HT autograft compared with BPTB autograft.

The restoration of symmetrical quadriceps and hamstring muscles strength and single-leg-hop test performance is a crucial goal of rehabilitation after ACLR [8, 17, 34]. Muscular asymmetries are known to be a risk factor for ACL graft tears and knee re-injuries $[8,17]$. Patients with a symmetrical isokinetic muscle strength and hop performance 6 months after ACLR have superior knee function and higher activity levels at the mid-term follow-up [31]. Quadriceps muscle strength is associated with patient-reported knee outcomes and satisfaction $[13,14,19,43]$, as well as with osteoarthritis development [22] after ACLR. Hamstring muscles strength deficits might be a risk factor for ACL re-tears, since they act as agonists to the ACL by resisting anterior tibial translation $[11,33]$.

The present study showed that the majority of patients are yet to regain symmetrical strength and function 6 months after ACLR. Increases in muscle volume and strength take a considerable length of time [39]. It is possible that, in most cases, 6 months are not sufficient to achieve symmetrical muscle strength and function after ACLR [34]. Even if recent studies suggested that RTS should not be permitted within 6 months after ACLR $[8,17]$, evaluating patients at this point provides clinicians with valuable information regarding patients' current knee function, their necessity for additional rehabilitation and their readiness to RTS [20]. Patients with identified knee function asymmetries, 6 months after ACLR, have time and could benefit from targeted interventions before their transition to a more complex rehabilitation and their gradually RTS. Overall, the most common deficit among the tests was found for isokinetic quadriceps strength. These results are in line with those in other investigations that have looked at isokinetic strength after ACLR [38, 40]. Rehabilitation protocols should be implemented and more time needs to be spent on muscle strength rehabilitation, especially for the quadriceps muscle.

The main strength of this study is the large sample size (4093 patients). This allowed a robust logistic regression analysis and a detailed study of several factors affecting the achievement of symmetrical knee strength and function after ACLR, such as all types of meniscal surgery, that have not been investigated in previous studies. All patients received surgery and isokinetic muscle strength and single-leg-hop test performance assessment at the same institution. Moreover, the study cohort represented a wide range of patients regarding age, time from injury to surgery, pre-injury activity level and concomitant meniscal surgery. The results of this study are, therefore, highly generalizable.

There are several limitations. First, even if a standardized rehabilitation protocol has been followed after ACLR, the study timeframe is long. Some small changes in rehabilitation 
could, therefore, have occurred over time. Second, information regarding preoperative muscle strength and knee pain during testing was not available. It has been shown that these variables are independently associated with quadriceps muscle strength 6 months after ACLR [36]. Third, there was no information regarding other hop tests that have been reported in previous studies $[8,9,16,17,20]$. These tests were not routinely employed at our institution for all patients.

The results of the present study have significant implications for the clinical management of patients after ACLR. To our knowledge, this is the first large cohort study which clearly shows that most patients do not achieve symmetrical knee function 6 months after primary ACLR. In addition, it provides unique data, comprising a detailed analysis of several factors affecting the achievement of symmetrical isokinetic muscle strength and single-leg-hop test performance after ACLR, which have not been investigated in previous studies. Every effort should be made to correct knee function asymmetries between the ACL-reconstructed knee and the healthy knee and the results of this study should be taken into consideration when planning rehabilitation after ACLR. With knowledge of the factors affecting the achievement of symmetrical knee function, rehabilitation protocols should be developed to be more customized/targeted, depending on patient characteristics, and therefore, more effective for every single patient.

\section{Conclusion}

Only $19.6 \%$ of the patients achieved symmetrical knee function 6 months after primary ACLR. Age $\geq 30$ years, MM resection and MM repair reduced the chance, whereas the use of HT autograft over BPTB autograft increased the chance of achieving symmetrical knee function 6 months after primary ACLR. This study shows that most of the patients are yet to regain symmetrical knee function 6 months after primary ACLR and, moreover, it identifies several factors affecting its achievement in a large cohort. The results of this study should be used to counsel patients about their expected functional recovery and to optimize rehabilitation and maximize knee function after ACLR.

Acknowledgements The authors thank Prof. Gunnar Edman from Karolinska Institutet for his invaluable help with the statistical analysis. The study received support from the Capio Research Foundation, the Sophiahemmet Research Foundation and the Eva \& Oscar Ahréns stiftelse.

Funding This study received funding for study purpose from the Capio research foundation, the Sophiahemmet research foundation and the Eva \& Oscar Ahrens stiftelse.

\section{Compliance with ethical standards}

Conflict of interest Each author declares that no possible conflict of interest (financial or nonfinancial) exist in connection with this study.
Ethical approval This study was approved from the Regional Ethics Committee, Karolinska Institutet, Diarie number: 2016/1613-31/2.

Informed consent For this type of study, formal consent is not required.

OpenAccess This article is distributed under the terms of the Creative Commons Attribution 4.0 International License (http://creativeco mmons.org/licenses/by/4.0/), which permits unrestricted use, distribution, and reproduction in any medium, provided you give appropriate credit to the original author(s) and the source, provide a link to the Creative Commons license, and indicate if changes were made.

\section{References}

1. Ardern CL, Webster KE, Taylor NF, Feller JA (2011) Return to sport following anterior cruciate ligament reconstruction surgery: a systematic review and meta-analysis of the state of play. Br J Sports Med 45(7):596-606

2. Barber SD, Noyes FR, Mangine RE, McCloskey JW, Hartman W (1990) Quantitative assessment of functional limitations in normal and anterior cruciate ligament-deficient knees. Clin Orthop Relat Res 255:204-214

3. Beynnon BD, Johnson RJ, Abate JA, Fleming BC, Nichols CE (2005) Treatment of anterior cruciate ligament injuries, Part I. Am J Sports Med 33:1579-1602

4. Duquin TR, Wind WM, Fineberg MS, Smolinski RJ, Buvea CM (2009) Current trends in anterior cruciate ligament reconstruction. J Knee Surg 22(1):7-12

5. Falconiero RP, DiStefano VJ, Cook TM (1998) Revascularization and ligamentization of autogenous anterior cruciate ligament grafts in humans. Arthroscopy 14(2):197-205

6. Farrell M, Richards JG (1986) Analysis of the reliability and validity of the kinetic communicator exercise device. Med Sci Sports Exerc 18:180-185

7. Gifstad T, Foss OA, Engebretsen L, Lind M, Forssblad M, Albrektsen G, Drogset JO (2014) Lower risk of revision with patellar tendon autografts compared with hamstring autografts: a registry study based on 45,998 primary ACL reconstructions in Scandinavia. Am J Sports Med 42(10):2319-2328

8. Grindem H, Snyder-Mackler L, Moksnes H, Engebretsen L, Risberg MA (2016) Simple decision rules can reduce reinjury risk by $84 \%$ after ACL reconstruction: the Delaware-Oslo ACL cohort study. Br J Sports Med 50(13):804-808

9. Hamrin Senorski E, Svantesson E, Beischer S, Thomeé C, Grassi A, Krupic F, Thomeé R, Karlsson J, Samuelsson K (2018) Concomitant injuries may not reduce the likelihood of achieving symmetrical muscle function one year after anterior cruciate ligament reconstruction: a prospective observational study based on 263 patients. Knee Surg Sports Traumatol Arthrosc 26(10):2966-2977

10. Heijne A, Hagströmer M, Werner S (2015) A two- and five-year follow-up of clinical outcome after ACL reconstruction using BPTB or hamstring tendon grafts: a prospective intervention outcome study. Knee Surg Sports Traumatol Arthrosc 23(3):799-807

11. Hiemstra LA, Webber S, MacDonald PB, Kriellaars DJ (2007) Contralateral limb strength deficits after anterior cruciate ligament reconstruction using a hamstring tendon graft. Clin Biomech (Bristol Avon) 22:543-550

12. Iriuchishima T, Shirakura K, Horaguchi T, Wada N, Sohmiya M, Tazawa M, Fu FH (2012) Age as a predictor of residual muscle weakness after anterior cruciate ligament reconstruction. Knee Surg Sports Traumatol Arthrosc 20(1):173-178

13. Ithurburn MP, Altenburger AR, Thomas S, Hewett TE, Paterno MV, Schmitt LC (2018) Young athletes after ACL reconstruction 
with quadriceps strength asymmetry at the time of return-to-sport demonstrate decreased knee function 1 year later. Knee Surg Sorts Traumatol Arthrosc 26(2):426-433

14. de Jong SN, van Caspel DR, van Haeff MJ, Saris DB (2007) Functional assessment and muscle strength before and after reconstruction of chronic anterior cruciate ligament lesions. Arthroscopy 23(1):21-28

15. Kramer J, Nusca D, Fowler P, Webster-Bogaert S (1993) Knee flexor and extensor strength during concentric and eccentric muscle actions after anterior cruciate ligament reconstruction using the semitendinosus tendon and ligament augmentation device. Am J Sports Med 21:285-291

16. Krych AJ, Woodcock JA, Morgan JA, Levy BA, Stuart MJ, Dahm DL (2015) Factors associated with excellent 6-month functional and isokinetic test results following ACL reconstruction. Knee Surg Sports Traumatol Arthrosc 23(4):1053-1059

17. Kyritsis P, Bahr R, Landreau P, Miladi R, Witvrouw E (2016) Likelihood of ACL graft rupture: not meeting six clinical discharge criteria before return to sport is associated with a four times greater risk of rupture. Br J Sports Med 50(15):946-951

18. Lautamies R, Harilainen A, Kettunen J, Sandelin J, Kujala UM (2008) Isokinetic quadriceps and hamstring muscle strength and knee function 5 years after anterior cruciate ligament reconstruction: comparison between bone-patellar tendon-bone and hamstring tendon autografts. Knee Surg Sports Traumatol Arthrosc 16:1009-1016

19. Lewek M, Rudolph K, Axe M, Snyder-Mackler L (2002) The effect of insufficient quadriceps strength on gait after anterior cruciate ligament reconstruction. Clin Biomech (Bristol Avon) 17(1):56-63

20. Logerstedt D, Grindem H, Lynch A, Eitzen I, Engebretsen L, Risberg MA, Axe MJ, Snyder-Mackler L (2012) Single-legged hop tests as predictors of self-reported knee function after anterior cruciate ligament reconstruction: the Delaware-Oslo ACL cohort study. Am J Sports Med 40(10):2348-2356

21. Nawasreh Z, Logerstedt D, Cummer K, Axe M, Risberg MA, Snyder-Mackler L (2018) Functional performance 6 months after ACL reconstruction can predict return to participation in the same preinjury activity level 12 and 24 months after surgery. Br J Sports Med 52(6):375

22. Øiestad BE, Juhl CB, Eitzen I, Thorlund JB (2015) Knee extensor muscle weakness is a risk factor for development of knee osteoarthritis. A systematic review and meta-analysis. Osteoarthr Cartil 23:117-171

23. Persson A, Fjeldsgaard K, Gjertsen JE, Kjellsen AB, Engebretsen L, Hole RM, Fevang JM (2014) Increased risk of revision with hamstring tendon grafts compared with patellar tendon grafts after anterior cruciate ligament reconstruction: a study of 12,643 patients from the Norwegian Cruciate Ligament Registry, 20042012. Am J Sports Med 42(2):285-291

24. Petschnig R, Baron R, Albrecht M (1998) The relationship between isokinetic quadriceps strength test and hop tests for distance and one-legged vertical jump tet following anterior cruciate ligament reconstruction. J Orthop Sports Phys Ther 28:23-31

25. Reid A, Birmingham TB, Stratford PW, Alcock GK, Giffin JR (2007) Hop testing provides a reliable and valid outcome measure during rehabilitation after anterior cruciate ligament reconstruction. Phys Ther 87(3):337-349

26. Richardson MS, Cramer JT, Bemben DA, Shehab RL, Glover J, Bemben MG (2006) Effects of age and ACL reconstruction on quadriceps gamma loop function. J Geriatr Phys Ther 29(1):28-34

27. Ross MD, Langford B, Whelan PJ (2002) Test-retest reliability of 4 single-leg horizontal hop tests. J Strength Cond Res 16(4):617-622

28. Samuelsson K, Andersson D, Karlsson J (2009) Treatment of anterior cruciate ligament injuries with special reference to graft type and surgical technique: an assessment of randomized controlled trials. Arthroscopy 25:1139-1174

29. Sànchez M, Anitua E, Azofra J, Prado R, Muruzabal F, Andia I (2010) Ligamentization of tendon grafts treated with an endogenous preparation rich in growth factors: gross morphology and histology. Arthroscopy 26(4):470-480

30. Sinaki M, Nwaogwugwu NC, Phillips BE, Mokri MP (2001) Effect of gender, age, and anthropometry on axial and appendicular muscle strength. Am J Phys Med Rehabil 80(5):330-338

31. Sousa PL, Krych AJ, Cates RA, Levy BA, Stuart MJ, Dahm DL (2017) Return to sport: Does excellent 6-month strength and function following ACL reconstruction predict midterm outcomes? Knee Surg Sports Traumatol Arthrosc 25(5):1356-1363

32. Tegner Y, Lysholm J (1985) Rating systems in the evaluation of knee ligament injuries. Clin Orthop Relat Res 198:43-49

33. Thomas AC, Villwock M, Wojtys EM, Palmieri-Smith RM (2013) Lower extremity muscle strength after anterior cruciate ligament injury and reconstruction. J Athl Train 48:610-620

34. Thomeé R, Kaplan Y, Kvist J, Myklebust G, Risberg MA, Theisen D, Tsepis E, Werner S, Wondrasch B, Witvrouw E (2011) Muscle strength and hop performance criteria prior to return to sport after ACL reconstruction. Knee Surg Sports Traumatol Arthrosc 19(11):1798-1805

35. Thomeé R, Neeter C, Gustavsson A, Thomeé P, Augustsson J, Eriksson B, Karlsson J (2012) Variability in leg muscle power and hop performance after anterior cruciate ligament reconstruction. Knee Surg Sports Traumatol Artrhosc 20:1143-1151

36. Ueda Y, Matsushita T, Araki D, Kida A, Takiguchi K, Shibata Y, Ono K, Ono R, Matsumoto T, Takayama K, Sakai Y, Kurosaka M, Kuroda R (2017) Factors affecting quadriceps strength recovery after anterior cruciate ligament reconstruction with hamstring autografts in athletes. Knee Surg Sports Traumatol Artrhosc 25(10):3213-3219

37. Undheim MB, Cosgrave C, King E, Strike S, Marshall B, Falvey E, Franklyn-Miller A (2015) Isokinetic muscle strength and readiness to return to sport following anterior cruciate ligament reconstruction: is there an association? A systematic review and a protocol recommendation. Br J Sports Med 49(20):1305-1310

38. Welling W, Benjaminse A, Seil R, Lemmink K, Zaffagnini S, Gokeler A (2018) Low rates of patients meeting return to sport criteria 9 months after anterior cruciate ligament reconstruction: a prospective longitudinal study. Knee Surg Sports Traumatol Arthrosc 26(12):3636-3644

39. Wernbom M, Augustsson J, Thomee R (2007) The influence of frequency, intensity, volume and mode of strength training on whole muscle cross-sectional area in humans. Sports Med 37(3):225-264

40. Wipfler B, Donner S, Zechmann CM, Jan Springer J, Siebold R, Paessler HH (2011) Anterior cruciate ligament reconstruction using patellar tendon versus hamstring tendon: a prospective comparative study with 9-year follow-up. Arthroscopy 27:653-665

41. Xergia SA, McClelland JA, Kvist J, Vasiliadis HS, Georgoulis AD (2011) The influence of graft choice on isokinetic muscle strength 4-24 months after anterior cruciate ligament reconstruction. Knee Surg Sport Traumatol Arthrosc 19:768-780

42. Yasuda K, Tsujino J, Ohkoshi Y, Tanabe Y, Kaneda K (1995) Graft site morbidity with autogenous semitendinosus and gracilis tendons. Am J Sports Med 23:706-714

43. Zwolski C, Schmitt LC, Quatman-Yates C, Thomas S, Hewett TE, Paterno MV (2015) The influence of quadriceps strength asymmetry on patient-reported function at time of return to sport after anterior cruciate ligament reconstruction. Am J Sports Med 43(9):2242-2249

Publisher's Note Springer Nature remains neutral with regard to jurisdictional claims in published maps and institutional affiliations. 